\title{
A new method to measure air-borne pyrogens based on human whole blood cytokine response
}

\author{
Ilona Kindinger ${ }^{\mathrm{a}}$, Mardas Daneshian ${ }^{\mathrm{a}}$, Hans Baur ${ }^{\mathrm{b}}$, Thomas Gabrio ${ }^{\mathrm{c}}$, \\ Andreas Hofmann ${ }^{\mathrm{d}}$, Stefan Fennrich ${ }^{\mathrm{a}}$, Sonja von Aulock ${ }^{\mathrm{a}}$, Thomas Hartung ${ }^{\mathrm{a}, \mathrm{e}, *}$ \\ ${ }^{a}$ Biochemical Pharmacology, University of Konstanz, POB M655, 78457 Konstanz, Germany \\ ${ }^{\mathrm{b}}$ Landwirtschaftliche Berufsgenossenschaft Baden-Württemberg, 70199 Stuttgart, Germany \\ ${ }^{\mathrm{c}}$ Landesgesundheitsamt Baden-Württemberg, Wiederholdstr. 15, 70174 Stuttgart, Germany \\ ${ }^{\mathrm{D}}$ Phytos, Leibnitzstr: 9, $89231 \mathrm{Neu}-U \mathrm{~lm}$, Germany \\ ${ }^{\mathrm{e}}$ EU Joint Research Center, ECVAM, 21020 Ispra, Italy
}

\begin{abstract}
Air-borne microorganisms, as well as their fragments and components, are increasingly recognized to be associated with pulmonary diseases, e.g. organic dust toxic syndrome, humidifier lung, building-related illness, "Monday sickness."

We have previously described and validated a new method for the detection of pyrogenic (fever-inducing) microbial contaminations in injectable drugs, based on the inflammatory reaction of human blood to pyrogens. We have now adapted this test to evaluate the total inflammatory capacity of air samples. Air was drawn onto PTFE membrane filters, which were incubated with human whole blood from healthy volunteers inside the collection device. Cytokine release was measured by ELISA.

The test detects endotoxins and non-endotoxins, such as fungal spores, Gram-positive bacteria and their lipoteichoic acid moiety and pyrogenic dust particles with high sensitivity, thus reflecting the total inflammatory capacity of a sample. When air from different surroundings such as working environments and animal housing was assayed, the method yielded reproducible data which correlated with other parameters of microbial burden tested. We further developed a standard material for quantification and showed that this assay can be performed with cryopreserved as well as fresh blood.

The method offers a test to measure the integral inflammatory capacity of air-borne microbial contaminations relevant to humans. It could thus be employed to assess air quality in different living and work environments.
\end{abstract}

Keywords: Air-borne pyrogens; Pyrogen test; Air quality

Abbreviations: CFU, colony-forming units; EEU, endotoxin equivalent units; EU, endotoxin units; IFN, interferon; IL, interleukin; IPT, in vitro pyrogen test; LAL, Limulus amoebocyte lysate assay; LPS, lipopolysaccharide; LTA, lipoteichoic acid; OD, optical density; PTFE, polytetrafluoroethylene; SEB, staphylococcal enterotoxin B.

* Corresponding author. Tel.: +49 7531 884116; fax: +49 7531884117.

E-mail address: Thomas.Hartung@uni-konstanz.de (T. Hartung). 


\section{Introduction}

Air-borne microorganisms of many different species are found in work and home environments. Inhaled bacterial and viral pathogens, organic dusts, components of the cell wall of Gram-negative bacteria (e.g. endotoxin, lipopolysaccharide), of Gram-positive bacteria (e.g. lipoteichoic acid), or of fungal spores, may have infectious, allergic and/or immunostimulatory consequences (Nowak, 1998; Schwartz, 2002).

They appear to contribute to organic dust toxic syndrome, chronic obstructive pulmonary disease, building-related illness, humidifier lung, or "Monday sickness". Epidemiological studies have shown that in occupational exposure the concentration of inhaled lipopolysaccharide (LPS) in bio-aerosols is strongly and consistently associated with reversible airflow obstruction (Kennedy et al., 1987; Schwartz et al., 1995; Milton et al., 1996).

Sampling and analysis of air quality is therefore the first step to determine whether the environment poses a potential threat to exposed people. Many different sampling methods and devices in use are based on impaction or filtration of air and the identification of live microorganisms by culturing. This latter method underestimates the total number of microbes in the air, since not all species grow on standard media. Moreover, culturing times differ and overgrowth of slowgrowing species is a problem. Another method is to determine the amount of dust in the air by gravimetric methods. However, although microbial cell wall components are known to be present in high concentrations in organic dust (Rylander et al., 1985), the dust weight gives no indication of biological activity. The Limulus amoebocyte lysate (LAL) assay specifically measures endotoxins of Gram-negative bacteria. Therefore, it reflects only a small part of the whole spectrum of air-borne microorganisms. Also, fungal glucans and DNA interfere with endotoxin detection in the LAL assay.

One major problem in this field is the lack of a standardized relevant methodology measuring total inflammatory burden and the lack of accepted threshold values. Thus, our aim was to develop a test method with an endpoint reflecting the human body's response to the complex mixture of compounds and organisms in a sample by means of a practicable and robust sampling and measuring methodology.
In 1995, we described a new method for the detection of pyrogenic (fever-inducing) substances (Hartung and Wendel, 1995). This whole blood assay utilizes the inflammatory response to pyrogens, including components of Gram-negative and Grampositive bacteria as well as fungi. Recognition of pyrogens by immune cells initiates the release of many signaling molecules, such as cytokines and eicosanoids. In the blood, monocytes are the main producers of the proinflammatory signal molecule interleukin-1 $\beta$ (IL-1 $\beta$ ) (Boneberg and Hartung, 2002), which changes the set-point for thermoregulation in the brain and causes a fever reaction in the organism. This is a very sensitive and conserved reaction. Cytokine release can be measured in vitro in the supernatant of human blood by an enzyme-linked immunosorbent assay (ELISA). Injectable drugs are the main area of application in which this test has recently been validated successfully in a collaborative European study (Hartung, 2002; Hoffmann and Hartung, 2005), the next step being incorporation into pharmacopoeias.

Here, we adapted the whole blood test to the measurement of air-borne pyrogens. Air was drawn through filters to collect the microbial contaminations using a pump, the filter membranes were then incubated with diluted human whole blood inside the collection device, and the release of IL- $1 \beta$ was analyzed after an overnight incubation. The air-sampling device, which was developed for this purpose, is a styreneacrylnitrile monitor system equipped with a polytetrafluoroethylene (PTFE) membrane filter (PALL Life Sciences, Ann Arbor, MI, USA). After air-sampling, the device can be closed and stored until measurement.

\section{Materials and methods}

\subsection{Air sampling}

Air was sampled with Personal Air Sampling Pumps (Model HFS-513 A, Gilian Sensidyne, Clearwater, USA, or Dräger accuro constant pump, Dräger Safety Schweiz AG, Dietlikon, Switzerland). Flowrates were between $0.21 / \mathrm{min}$ and $3.51 / \mathrm{min}$ as indicated. For multiple measurements with one pump, 5 polypropylene tubes were connected to the pump 
and the air flow was regulated by flowmeters (Key Instruments, Trevose, USA) adjustable between 0.1 and 1 1/min (Fig. 1). Air monitor cassettes of styreneacrylnitrile with a diameter of $37 \mathrm{~mm}$ with polytetrafluoroethylene filters (especially manufactured and generously provided by PALL Life Sciences, Ann Arbor, USA) were used for collection. Dust samples were collected with a photometric particle sampler (Respicon TM, Hund, Wetzlar, Germany).

\subsection{Whole blood incubation}

Differential blood cell counts were routinely performed with a Pentra 60 (ABX Diagnostics, Montpellier, France) to exclude acute infections of blood donors. $300 \mu 1$ or $500 \mu 1$ of heparinized blood freshly taken from healthy volunteers was diluted 12 fold with isotonic saline solution (Berlin-Chemie AG,
Berlin, Germany) and incubated overnight at $37{ }^{\circ} \mathrm{C}$ without agitation according to the protocol of the Endosafe-IPT kit (Endosafe-IPT kit, Charles River Endosafe, Charleston, USA). Initial control experiments with the polytetafluorethylene filters (PALL Life Sciences) were performed in Petri dishes in a total incubation volume of $6000 \mu$.

Whole blood incubations in air monitor cassettes were carried out in a total volume of $3600 \mu$. The IL$1 \beta$ response to the filters contaminated with air samples was compared with the response to a concentration-response curve to lipopolysaccharide (LPS) without filters, or to unused filters plus known concentrations of LPS added to the incubation, and to the basal response induced by clean filters.

The control stimuli, LPS from Escherichia coli strain O-113 (WHO reference material, a generous gift from Dr. S. Poole, National Institute for Bio-

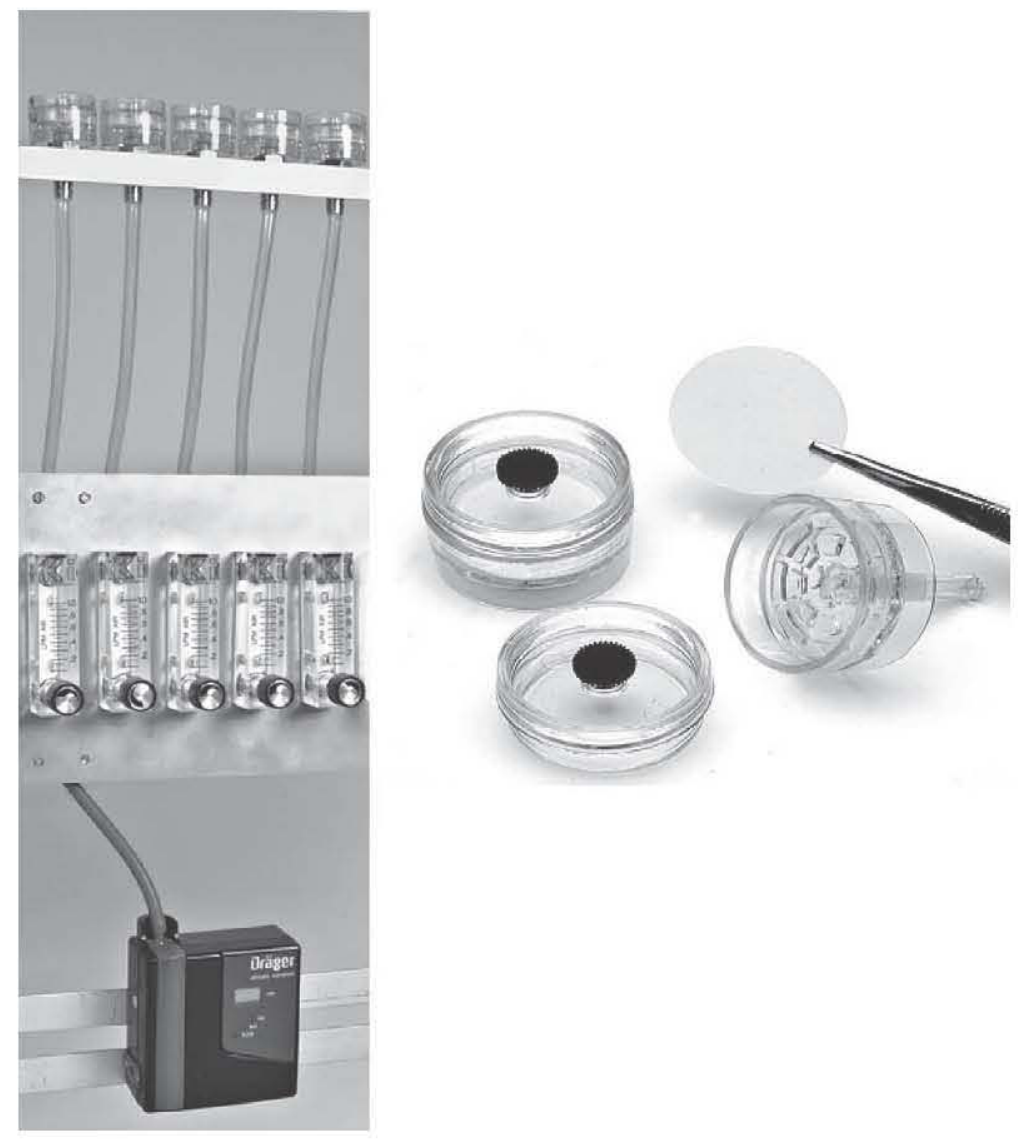

Fig. 1. Air-sampling set-up and sampling monitor. 
logical Standards and Controls, Hertfordshire, UK) or LTA from Bacillus subtilis (prepared in-house according to Morath et al., 2001) and provided in the Endosafe-IPT kit were applied to the filter membranes in a volume of a 12 th of the total incubation volume, i.e. 300 or $500 \mu 1$. Incubations were carried out in the presence of $5 \% \mathrm{CO}_{2}$ at $37{ }^{\circ} \mathrm{C}$ overnight. Blood suspensions were then transferred to $14 \mathrm{ml}$ polystyrene tubes (Greiner, Germany). Cell-free supernatants were obtained by centrifugation at $3500 \times \mathrm{g}$ for $2 \mathrm{~min}$ and used for cytokine determination immediately, or stored at $-80{ }^{\circ} \mathrm{C}$.

To measure lymphokine release after 48 or $72 \mathrm{~h}$ incubations, blood was diluted in RPMI 1640 (Bio Whittaker, Verviers, Belgium) instead of saline. The superantigen staphylococcal enterotoxin B (SEB, Sigma, Deisenhofen, Germany) was used as control stimulus here (Hermann et al., 2003).

To test the inflammatory response to fungal spores, $1000 \mu \mathrm{l}$ isotonic saline were pipetted into a $1.5 \mathrm{ml}$ polypropylene reaction tube (Eppendorf, Hamburg, Germany) and $100 \mu \mathrm{l}$ fungal spore suspension or LPS and $100 \mu \mathrm{l}$ whole blood were added and incubated as described. Fungal spores from the different species were obtained in suspensions from T.G., Landesgesundheitsamt Baden-Württemberg, Stuttgart, Germany. The spore cultures were inoculated on sterile polycarbonate filters and cultured on malt-agar plates.

After incubation, the tubes were shaken and blood cells were sedimented by centrifugation $(16000 \times g$, $2 \mathrm{~min}$ ).

In some experiments polymyxin B (Sigma-Aldrich, Deisenhofen, Germany) was added to a final concentration of $5 \mu \mathrm{g} / \mathrm{ml}$.

\subsection{Incubations with cryopreserved blood}

Cryopreserved blood was prepared as described (Schindler et al., 2004). Briefly, the heparinized blood was pre-cooled in ice water for $15 \mathrm{~min}$. Clinical grade dimethylsulfoxide (DMSO, Waco Chemicals, DessauThornau, Germany) was added to the blood in $50 \mathrm{ml}$ centrifugation tubes (Greiner bio-one, Frickenhausen, Germany) in small amounts to a final concentration of $10 \%$ under constant gentle agitation. Blood was pipetted as $4 \mathrm{ml}$ aliquots into pre-cooled cryotubes $(1.8,3.6$ or $4.5 \mathrm{ml}$, Nunc, Wiesbaden, Germany) and put into the rack of a pre-cooled to $4{ }^{\circ} \mathrm{C}$ Nicool Plus PC (Air Liquide, Marne-la-Vallée Cedex 3, France). The blood was cooled down to $-5{ }^{\circ} \mathrm{C}$ at a rate of 1 ${ }^{\circ} \mathrm{C} / \mathrm{min}$. The temperature $\mathrm{Tx}$ in the freezing chamber was set to $-30{ }^{\circ} \mathrm{C}$. The crystallization temperature was $-12{ }^{\circ} \mathrm{C}$. When this temperature was reached, the blood was cooled down to $-40{ }^{\circ} \mathrm{C}$ at a rate of $2{ }^{\circ} \mathrm{C} /$ $\min$. The blood was given $120 \mathrm{~s}$ to stabilize before being cooled down to $-120^{\circ} \mathrm{C}$ at a rate of $10^{\circ} \mathrm{C} / \mathrm{min}$. After freezing, the tubes were removed from the freezer and put immediately into the vapor phase of liquid nitrogen (nitrogen tank, Air Liquide, Kryotechnik, Düsseldorf, Germany). After thawing for $30 \mathrm{~min}$ at $37{ }^{\circ} \mathrm{C}$, the blood was used as described above for fresh blood without washing steps to remove the DMSO.

\subsection{ELISA}

IL-1 $\beta$ was measured with Endosafe-IPT (In Vitro Pyrogen Test kindly provided by Charles River Laboratories, Charleston, USA) according to the manufacturer's instructions. Other cytokine measurements were done with sandwich ELISAs, based on antibody pairs against human IL-1 $\beta$, IL-6 (both R\&D Systems, Wiesbaden, Germany), IL-8, IFN- $\gamma$ (both Endogen, Eching, Germany) and IL-5 (BD Biosciences, Heidelberg, Germany). Recombinant cytokines serving as standards (IL-1 $\beta, \mathrm{IL}-6$ and IL-8) were gifts from Dr. S. Poole (National Institute for Biological Standards and Controls). Standards for IFN- $\gamma$ and IL-5 were obtained from Endogen and BD Biosciences, respectively.

\subsection{Limulus assay}

The kinetic chromogenic Limulus amoebocyte lysate assay (LAL) was performed according to the manufacturer's instructions (Endochrome-K, Charles River Endosafe). After air sampling, the filter material (either PTFE or glass fiber) was rinsed with $10 \mathrm{ml}$ pyrogen-free water containing $0.05 \%(\mathrm{v} / \mathrm{v})$ polyoxyethylenesorbitan-monolaurate (Tween 20). Filters were shaken for $60 \mathrm{~min}$ on an orbital shaker at room temperature. The extract was centrifuged at $1000 \times g$ for $10 \mathrm{~min}$. One clean filter was treated in the same way as a negative control. $100 \mu \mathrm{l}$ of the supernatant were employed in the LAL and the obtained value 
was multiplied by 100 to obtain the endotoxin per 10 $\mathrm{ml}$ sample.

\subsection{Sampling of bacteria and fungi}

For determination of air-borne bacteria and fungi, samples were drawn with an impaction air sampler SAS Super 90 (International pbi S.p.A., Milano, Italy) with either CASO agar plates or Sabouraud- $4 \%$ glucose-agar plates (Merck, Darmstadt, Germany) with $50 \mathrm{mg} / 1$ chloramphenicol (Sigma-Aldrich, Taufkirchen, Germany) attached. The sampling volume was 1001 for outside air and 101 for indoor air. The Sabouraud plates were incubated at $25^{\circ} \mathrm{C}$ for $3-5$ days, the CASO plates at $37^{\circ} \mathrm{C}$ for $3-5$ days. The colony counts were corrected with a correction table to adjust colony counts for the Impactor as instructed before calculating the final colony forming units (CFU) per $\mathrm{m}^{3}$.

\subsection{Gram-staining}

$100 \mu \mathrm{l}$ of $500 \mu \mathrm{g} / \mathrm{ml}$ dust suspensions of ground dust material (obtained by sampling dust in different pig pens and fractionized with a fluidized opposed bed jet mill with a grind pot diameter of $200 \mathrm{~mm}$ and 6 bar pressure, Sympatec GmbH, Augsburg, Germany) were spread on blood agar plates (Columbia, Heipha, Heidelberg, Germany) and incubated at $37^{\circ} \mathrm{C}$ for 3 days. After counting the $\mathrm{CFU}$, single representative colonies of each morphotype were picked and smeared on slides. After drying, the smears were heatfixed with a Bunsen flame. Gram-staining was done with a Gram-staining set (Merck) according to the manufacturer's instructions and evaluated by microscopic examination.

\subsection{Development of a standard material}

Dust from various pig pens was collected, by dusting rafters. Dust grains from a sample of this material were fractionated by size and a sample was ground to a particle size smaller than $10 \mu \mathrm{m}$ ( $90 \%$ of sample) that represents inhalable particles. The endotoxin content of the dust material in suspension was $174 \mathrm{EU} / \mathrm{mg}$ as determined by LAL. In the whole blood assay we determined a signal equivalent to $150 \mathrm{EU} / \mathrm{ml}$. This value was calculated from dose-response curves of 8 donors, by comparison with the reaction towards LPS from $E$. coli O-113.

When the dust suspension was spread on blood agar plates and incubated at $37^{\circ} \mathrm{C}$, many different bacterial $\left(3.5 \times 10^{3} \mathrm{CFU} / \mathrm{mg}\right.$ dust $)$ and fungal colonies were counted $\left(4 \times 10^{3} \mathrm{CFU} / \mathrm{mg}\right.$ dust). Most of the bacterial CFU were Gram-negative rods, but we also found some Gram-positive coccoid forms. Pasteurization $\left(80^{\circ} \mathrm{C}\right.$ for $\left.10 \mathrm{~min}\right)$ reduced the number of CFU to a 10 th. In the presence of the antimycotic amphotericin B only one bacterial strain grew with a large number of colonies, while no fungi grew. In the presence of the antibiotics penicillin and streptomycin 3 to 5 fungal colonies and 2 to $4 \mathrm{P} / \mathrm{S}$ resistant bacterial strains grew.

\subsection{Statistics}

One-way ANOVA with Dunnet's post test was performed using GraphPad Prism 3.0 (GraphPad Software, San Diego, CA, USA). * $p<0.05 ; *^{*} p<0.01$.

\section{Results}

\subsection{Suitability of filter material}

To adapt the IPT to the measurement of air samples, we required a pyrogen-free material with no interference in the whole blood test. Different concentrations of LPS from $E$. coli and LTA from B. subtilis were applied to the polytetrafluoroethylene (PTFE) filters before incubation with whole blood. The IL- $1 \beta$ response to LPS or LTA in the presence of these filter materials was compared with the response of the blood to LPS or LTA without filter material in the same experiment. The hydrophobic PTFE showed no basal cytokine induction and did not interfere with the pyrogen-induced response to the control stimuli. As endotoxin accumulation is expected at particle sizes between 3.5 and $8.5 \mu \mathrm{m}$ (Attwood et al., 1986), the pore size $5 \mu \mathrm{m}$ was chosen since this would not block as readily as smaller sizes. These filters were inserted into the air-monitor cassettes on top of a watertight cellulose support pad supplied by PALL Life Sciences. 


\subsection{Field measurements}

Air samples from animal housing were taken in field measurements, as the contamination of air in such locations is especially problematic for people working there (Nowak, 1998).

Data from air samples from a cattle shed, showed a clear correlation with the sampling time and a good reproducibility with a c.v. of $11 \%$ to $15 \%$ (Fig. 2). Compared to a dose-response curve of LPS, the sampling time of $5 \mathrm{~min}$ showed IL- $1 \beta$-inducing activity equivalent to $83 \mathrm{pg}$ LPS, i.e. $0.83 \mathrm{EU} .1 \mathrm{EU}$ is defined as $100 \mathrm{pg}$ of the WHO reference LPS from E. coli $\mathrm{O}-113$. Thus, endotoxin equivalent unit (EEU) means IL- $1 \beta$ release equivalent to that in response to the reference LPS in EU. It should be noted that the comparison to the cytokine induction by LPS does not indicate how much LPS is contained in the sample as other potent pyrogens, such as structures from Grampositive bacteria or from fungal spores likely make up a significant proportion of the inflammatory capacity of the air sample.

The value after $15 \mathrm{~min}$ of sampling is not higher than after $10 \mathrm{~min}$. This implies that some form of saturation does eventually take place. This may result from saturation of the filters, saturation of the blood cells or saturation of the ELISA. These limitations of the test will need to be defined more closely in further experiments to set guidelines for the measurement in different environments.

We followed the time course of 1 day in a calf shed to correlate the inflammatory potential with the CFU counts and working conditions (Fig. 3). We found a good correlation between $\mathrm{CFU} / \mathrm{m}^{3}$ and the $\mathrm{IL}-1 \beta$ signal with the highest signals during working periods.

We then performed similar comparative measurements in different animal housing and at working places in which high concentrations of microbes were expected (Table 1). Pig pen 1 had wooden boards with gaps in between. Pig pen 2 was a traditional structure with straw on earthen ground. Further measurements were done in a calf shed and a plastic recycling plant, where rubbish is screened for reusability by hand. The samples were drawn next to a conveyor belt. As can be seen in Table 1 , the IL-1 $\beta$ release corresponded better to the dust concentrations than to the measured CFU. This can be explained by the fact that the immune stimulatory potency of the different germs varies from species to species, that not all bacteria grow on standard agar plates, and that the presence of non-living inflammatory material is neglected in the CFU determinations.

Airborne fungal contaminations, both the spores themselves and their toxic metabolites pose a health hazard for humans (Fischer and Dott, 2003). Using

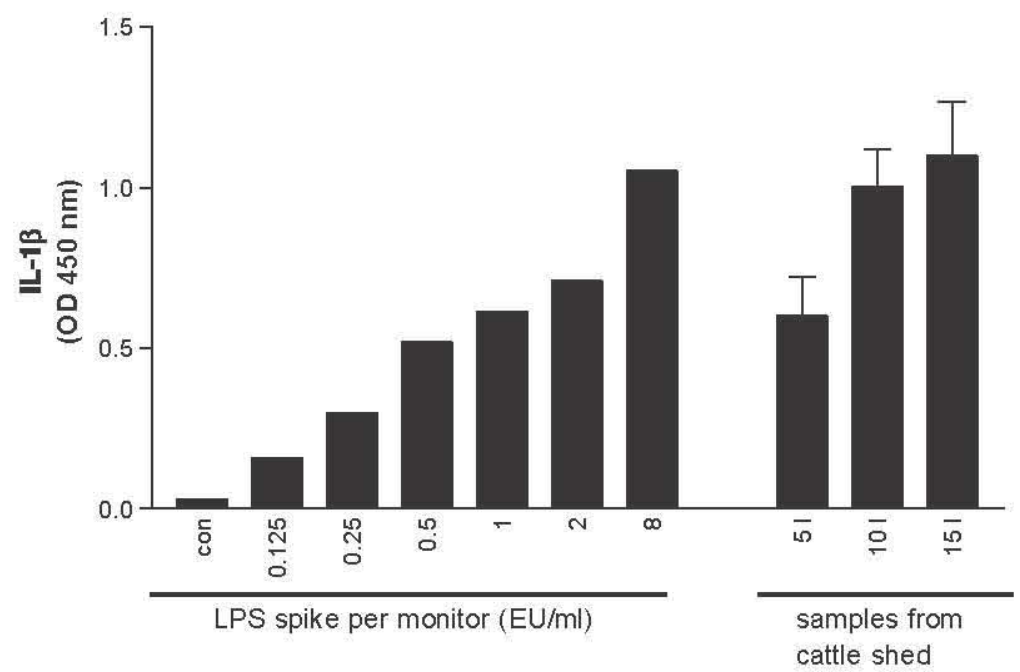

Fig. 2. Release of IL-1 $\beta$ in human whole blood induced by defined LPS concentrations on PTFE membranes in air sampling monitors ( $n=1$, left half) or contaminated in a cattle shed ( $n=5 \pm$ S.D.) by collecting 5,10 and $15 \mathrm{l}$ at $1 \mathrm{l} / \mathrm{min}$ (right half). 


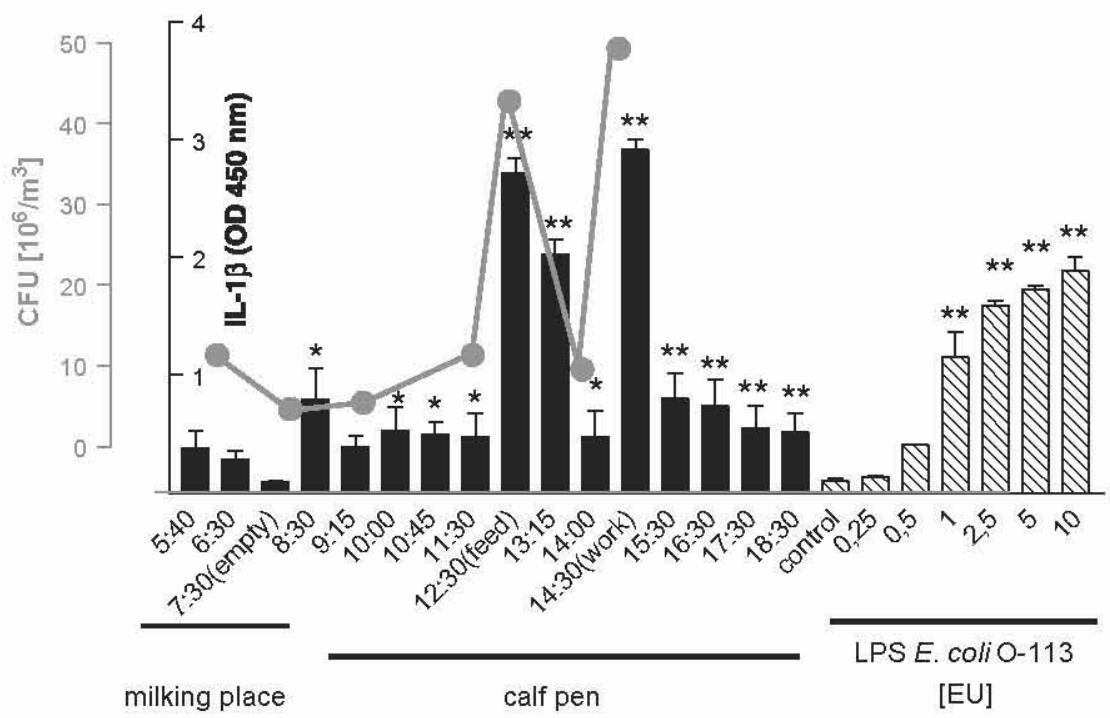

Fig. 3. Air-sampling over 1 day at different places on a farm. Air-sampling was carried out in air monitor cassettes in 5-fold values for each time point. Sampling volume was $5 \mathrm{l}$ at $1 \mathrm{l} / \mathrm{min}$. Colony forming units were determined by counting colony growth after sampling air (10 l) at the indicated times. ${ }^{*} p<0.05 ;{ }^{*} p<0.01$ vs. control.

cultures of four common airborne fungal spore species (Alternaria alternata, Penicillium crustosum, Cladosporium cladosporoides, Aspergillus versicolor) we could demonstrate that they are also detected dosedependently in the IPT. The cytokine response to the fungal spores was not inhibited by polymyxin B. Fungal glucan is known to interfere with the lysate of the Limulus assay, giving false-positive results. When the $\beta$-glucan pathway was blocked with an endotoxinspecific-buffer BG 120 containing carboxymethylated curdlan (Charles River Endosafe), the spores resulted in no detectable signals in the LAL. We verified this result by inhibiting potential LPS signaling with polymyxin $\mathrm{B}$.

Table 1

Release of $\mathbb{L}-1 \beta$ in human whole blood induced in response to air samples from different surroundings

\begin{tabular}{lclc}
\hline Location $^{\text {a }}$ & EEU/ml \pm S.D. & mg dust $/ \mathrm{m}^{3 \mathrm{c}}$ & $\mathrm{CFU} / \mathrm{m}^{3}$ \\
\hline Pig pen 1 & $4.6 \pm 0.17$ & 0.9 & 17000 \\
Pig pen 2 & $16 \pm 0.12$ & 1.5 & 50000 \\
Calf shed & $0.9 \pm 0.12$ & 0.5 & 14000 \\
Recycling plant & $0.2 \pm 0.22$ & 0.1 & 7000 \\
\hline
\end{tabular}

\footnotetext{
a Sampling volume $5 \mathrm{l}$ at $1 \mathrm{l} / \mathrm{min}$.

b Means of 5 -fold measurements \pm S.D.

${ }^{\circ}$ Dust and CFU were determined in parallel.
}

\subsection{Comparison of IPT with LAL}

We compared the measurement of air samples drawn in parallel in the whole blood assay and in the Limulus amoebocyte lysate assay (LAL). As the LAL can only be used in clear fluids, not on a solid phase material, samples had to be rinsed from the monitor and the obtained values were multiplied by the dilution factor. For this reason, it is not possible to control by LAL how well the sample has been solubilised from the collection material. The IPT allows direct contact of the blood with the sample. Air samples were collected in a cattle shed and a pig pen. As was expected, the values determined in comparison to the same reference LPS were lower in the LAL by a factor of 2 to 25 , illustrating the difference between the endotoxin content and the total inflammatory capacity relevant to humans detected by the whole blood system. In addition, the solubilisation of the sample for the LAL measurement may have variable efficiency depending on the material used for collection and the rinsing conditions. However, this cannot be controlled with this method. Therefore, the supernatant does not necessarily represent the entire spectrum of collected air-borne particles. In the 
whole blood test, however, the monocytes are able to reach even particles which are embedded in the filter pores, as they are in direct contact with the filter material.

To confirm these results, we collected air in a cattle and a pig pen. After sampling 4 monitors in parallel, one filter membrane was transferred from the monitor into a sterile Petri dish and washed with $10 \mathrm{ml}$ pyrogen-free water containing Tween 20. After shaking for $1 \mathrm{~h}$ and centrifugation, as required for LAL measurements, the wash solution was employed in the LAL assay and for comparison in the IPT. The washed filter was transferred to a new air-sampling monitor and also tested in the IPT to determine the effectiveness of the washing procedure. Three monitors with unwashed filters were measured directly in the IPT (Table 2). The measurement of the lavage in both assays shows increasing values between 5 and $30 \mathrm{~min}$ sampling time in both cases, although the results in the IPT appear lower. This may be explained by the presence of fungal glucans in the samples, which give a false-positive result in the LAL. More importantly, the washed filters still give high signals in the IPT, indicating that the rinsing procedure only washes a small amount of the pyrogen from the filter. Thus the advantage of the IPT system lies not only in its ability to react to more than endotoxin from Gram-negative bacteria, but also in the fact that the direct contact between filter sample and blood results in a more representative measurement.

\subsection{Development of a standard material}

As LPS contributes only a part of the total inflammatory capacity of an air sample, we produced a standard dust material as a more relevant comparison. The cytokine response to the different particle sizes of the dust showed similar potencies in all fractions in comparison to the original, unground as well as the ground material (Fig. 4). Therefore, the entire dust was ground to $90 \%<10 \mu \mathrm{m}$. This dust was now characterized in terms of comparing the cytokine profile induced in blood to that of LPS. The kinetics of cytokine induction by dust were similar to those induced by LPS. IL- 8 was secreted after 2 h, IL-1 $\beta$ and IL- 6 after $4 \mathrm{~h}$ of incubation. Release of IFN- $\gamma$ was measured after $48 \mathrm{~h}$ while IL-5 induction was not detectable, even after $72 \mathrm{~h}$ incubation (control stimulus in this case was SEB).

\subsection{Use of cryopreserved instead of fresh blood}

The major concerns about using human blood for this type of test are individual differences of donors, availability of blood and possible threat of bloodtransmitted infections. These have recently been overcome by the development of a procedure to cryopreserve blood, allowing pooling of blood from different donors, pre-testing reactivity as well as ruling out typical infectious risks (Schindler et al., 2004). Here we show, that the dose-response curves for LPS (Fig. 5A) and the ground dust material (Fig. 5B) had similar shapes for fresh and cryopreserved

Table 2

Comparison of air samples taken in a calf shed and a pig pen

\begin{tabular}{|c|c|c|c|c|}
\hline \multirow{2}{*}{$\begin{array}{l}\text { Place of measurement } \\
\text { Sampled air volume }\end{array}$} & \multicolumn{3}{|c|}{ Calf shed ${ }^{a}$} & \multirow{2}{*}{$\frac{\text { Pig pen }^{\mathrm{b}}}{11}$} \\
\hline & 51 & $10 \mathrm{I}$ & 301 & \\
\hline Lavage tested in $\mathrm{LAL}^{\circ}$ & 0.9 & 7.5 & 19 & 1.6 \\
\hline Lavage tested in IPT & 2.1 & 4.5 & 6 & 1.4 \\
\hline Filter in monitor tested in IPT & $9 \pm 0.06$ & $16 \pm 0.05$ & $>50 \pm 0.12$ & $42 \pm 0.14$ \\
\hline Washed filter in monitor tested in IPT & 3 & 1 & $>50$ & 31 \\
\hline
\end{tabular}

\footnotetext{
${ }^{a}$ Drawn at $1 \mathrm{l} / \mathrm{min}$.

${ }^{b}$ Drawn at $0.2 \mathrm{l} / \mathrm{min}$.

c Monitors were sampled in parallel; three were assayed directly in the IPT, the filter of one was extracted as required for the LAL assay. The lavage was tested in LAL and IPT. Of the total lavage volume $(10 \mathrm{ml}), 100 \mu \mathrm{l}$ were employed in both test systems and results calculated for total sample. Filters in monitors were assayed in the IPT (values \pm SD). Controls of the lavage solution without a filter and lavage with a clean filter were carried out but gave no significant response in either test system. EEU values per sample were calculated based on the same reference LPS used in both test systems.
} 


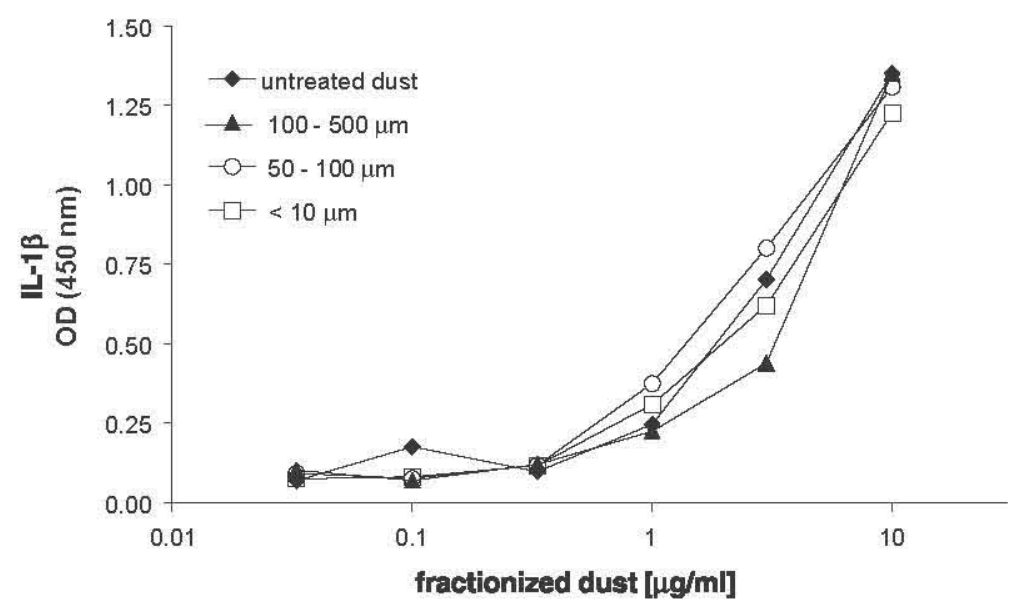

Fig. 4. Dust fractions of different particle size from a pig pen induce $\mathbb{L}-1 \beta$ release in human whole blood, mean of double values.
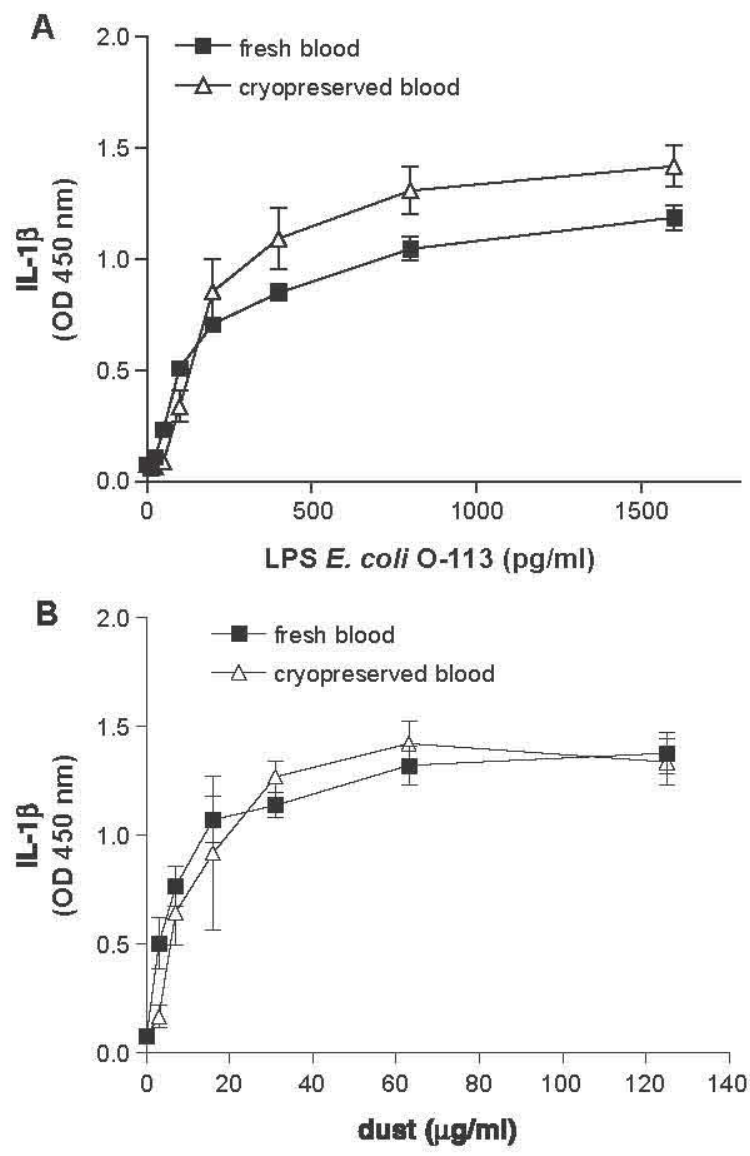

Fig. 5. Fresh and cryopreserved human whole blood releases comparable amounts of $\amalg-1 \beta$ in response to LPS from $E$. coli strain O-113 (A) and pig dust particles (B), mean of 4 blood donors \pm S.E.M. blood, indicating that the assay can also be performed with cryopreserved blood.

3.6. Do persons allergic to house dust and mites represent suitable blood donors?

The cytokine response of blood from five donors diagnosed as allergic to dust and/or mites by a prick test but not in a hypersensitive phase or on antihistaminic medication was compared to blood from five donors with no such allergic history. The differential blood cell counts showed no difference between the groups regarding eosinophil count, confirming that the donors were not hypersensitized. This blood was exposed to various concentrations of LPS, pigpen dust or samples collected from a cattle shed. The allergic donors tended towards slightly, though not significantly, higher IL-1 $\beta$ release in response to each of the stimuli over the dose-response curves (Fig. 6). Thus, persons with known allergies towards air-borne allergens should probably be excluded as blood donors for the test. This can easily be achieved by the provision of pretested, cryopreserved blood.

\section{Discussion}

To develop a new method to evaluate the total inflammatory potential of air samples, we needed to find a relevant endpoint, establish a suitable sampling 

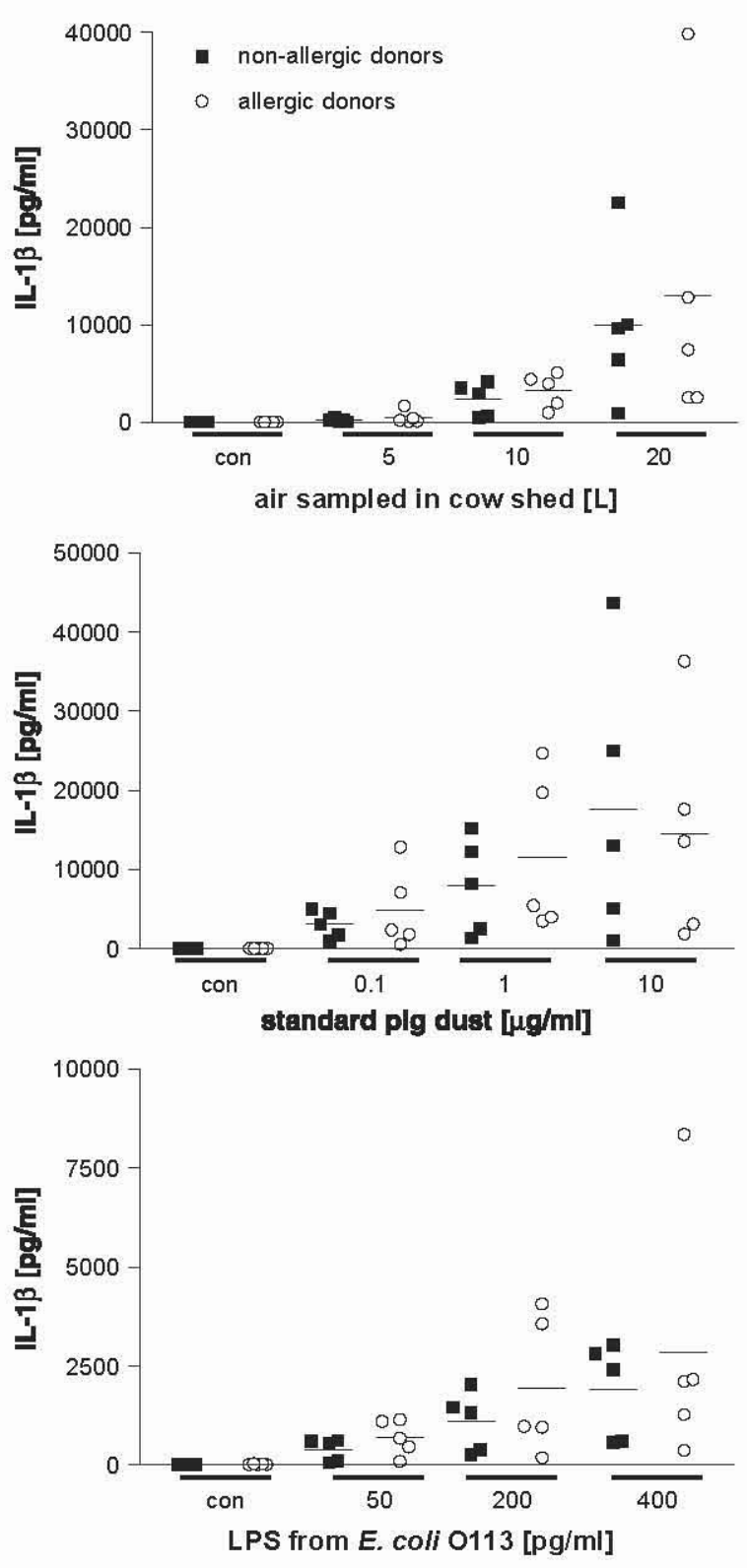

Fig. 6. Similar response of blood from donors allergic to house dust and mites (black bars) and blood from non-allergic donors (white bars) to various concentrations of LPS, pig dust and air samples from a cow shed, mean of 5 blood donors \pm S.E.M.

method, determine whether the detection spectrum encompasses all likely targets, develop a standard or reference material and perform initial field studies.

The basic strategy of the human whole blood assay (IPT) is to determine pyrogenic contaminations relevant to humans by exploiting the endogenous defense reaction to these substances. Thus, we decided to adapt this test for the purpose of evaluating the total inflammatory capacity of the microbial burden in air. Sampling with the monitors proved practicable as they could be closed, stored and transported after collection. Moreover, as the incubation could be performed directly inside the monitors the filters did not have to be handled. Apart from immune stimuli already well characterized in this system, i.e. LPS from Gram-negative and LTA from Gram-positive bacteria, we showed that the test sensitively detects common air-borne fungal spores in a dose-dependent manner. As a highly purified endotoxin standard is not ideally suited for comparison with highly complex air samples, the pig dust was collected and characterized as a reference material. The field studies performed showed the feasibility of the approach giving correlations with, but at the same time highlighting differences from, the microbiological culture or the LAL. Finally, the difficulty of obtaining standardized and pretested blood to perform the assay was dispelled by showing that cryopreserved blood can be used instead of fresh blood. Also, as a precaution, only blood from donors with no known allergies towards air-borne allergens should be used. To the best of our knowledge there is no comparable test system that can cover dead and living material in an integral manner.

The highly effective uptake of inhaled substances by the $100 \mathrm{~m}^{2}$ of mucous membrane in the lung results in effects similar to injection of these substances. Safety measurements in workplaces and correlation with environmental hazards and their consequences for human health are therefore important both to identify problematic areas and to judge the effectiveness of mitigating measures. The key remaining problem in judging the risk is the lack of accepted threshold values and the lack of representative methods to measure the exposure. As seen by the comparison of the LAL with the IPT measurement, the LAL is not only limited to the measurement of endotoxin but also is likely severely limited by requiring a clear fluid for measurement, i.e. solids must be rinsed and measurements performed in the rinsing solution. This result has serious implications for other applications of the LAL, such as for the safety testing of medical devices and for other uses of 
rinsing solutions of solid bodies, such as the rabbit pyrogen test for medical devices.

The crucial advantage of this new test is that the model mirrors the cytokine reaction that the total burden would evoke in the blood of humans, thereby reflecting the potential danger to humans. However, it cannot reflect the entire spectrum of immune reactions that might be triggered in the lung, i.e. mucosal immune reactions, responses of the adaptive immune system, etc.

Further developments and standardizations of the test will entail the determination of the volume of air required for a representative sample under various conditions, definition of controls and reference materials to allow (semi-)quantitative analysis of the air load as well as the definition of the number of replicates required for the different procedural steps as quality controls. It would possible to measure the reaction of the blood of exposed persons towards samples from their environment, thus also taking possible sensitization or other individual factors into account. Here again, the use of pretested, standardized cryopreserved blood from non-allergic donors as a comparison would be valuable. A whole blood pyrogen test based on animal blood (Schindler et al., 2003 ) could be developed to estimate the inflammatory reaction of animals living in a pen or shed and to compare their sensitivity to that of humans.

We intend to evaluate this innovative method further with partners from environmental and occupational medicine, work safety and industry to optimize the methodological developments described for the specific areas of application.

The new test system developed here opens the opportunity to monitor exposures to inhalable inflammatory stimuli in an integrative manner for the first time. Following the standardization undertaken here, epidemiological studies will allow assessment of the contribution of air-borne pyrogens to various pulmonary diseases.

\section{Acknowledgments}

This work was supported by the Bundesministerium für Wirtschaft und Technologie, Germany, Grant KF 0315701 KRF1. A patent (T.H.) of the method is pending.

\section{References}

Attwood, P., Versloot, P., Heederik, D., de Wit, R., Boleij, J.S., 1986. Assessment of dust and endotoxin levels in the working environment of Dutch pig farmers: a preliminary study. Ann. Occup. Hyg. 30, 201.

Boneberg, E.M., Hartung, T., 2002. Granulocyte colony-stimulating factor attenuates LPS-stimulated $\mathrm{L}$-1 beta release via suppressed processing of proIL-1beta, whereas TNF-alpha release is inhibited on the level of proTNF-alpha formation. Eur. J. Immunol. 32, 1717.

Fischer, G., Dott, W., 2003. Relevance of airborne fungi and their secondary metabolites for environmental, occupational and indoor hygiene. Arch. Microbiol. 179, 75.

Hartung, T., 2002. Comparison and validation of novel pyrogen tests based on the human fever reaction. Altern. Lab. Anim. 30 (Suppl. 2), 49.

Hartung, T., Wendel, A., 1995. Detection of pyrogens using human whole blood. Altex 12, 70 .

Hermann, C., von Aulock, S., Graf, K., Hartung, T., 2003. A model of human whole blood lymphokine release for in vitro and ex vivo use. J. Immunol. Methods 275, 69 .

Hoffmann, S., Hartung, T., 2005. Diagnosis: toxic!-Trying to apply approaches of clinical diagnostics and prevalence in toxicology considerations. Toxicol. Sci. (electronic publication ahead of print, Feb. 2).

Kennedy, S.M., Christiani, D.C., Eisen, E.A., Wegman, D.H., Greaves, I.A., Olenchock, S.A., Ye, T.T., Lu, P.L., 1987. Cotton dust and endotoxin exposure-response relationships in cotton textile workers. Am. Rev. Respir. Dis. 135, 194.

Milton, D.K., Wypij, D., Kriebel, D., Walters, M.D., Hammond, S.K., Evans, J.S., 1996. Endotoxin exposure-response in a fiberglass manufacturing facility. Am. J. Ind. Med. 29, 3.

Morath, S., Geyer, A., Hartung, T., 2001. Structure-function relationship of cytokine induction by lipoteichoic acid from Staphylococcus aurets. J. Exp. Med. 193, 393.

Nowak, D., 1998. Health effects of airborne pollutants, particularly in swine confinement stalls, from the viewpoint of occupational medicine. Dtsch. Tierarztl. Wochenschr. 105, 225.

Rylander, R., Haglind, P., Lundholm, M., 1985. Endotoxin in cotton dust and respiratory function decrement among cotton workers in an experimental cardroom. Am. Rev. Respir. Dis. 131, 209.

Schindler, S., Bristow, A., Cartmell, T., Hartung, T., Fennrich, S., 2003. Comparison of the reactivity of human and rabbit blood towards pyrogenic stimuli. Altex 20, 59 .

Schindler, S., Asmus, S., von Aulock, S., Wendel, A., Hartung, T., Fennrich, S., 2004. Cryopreservation of human whole blood for pyrogenicity testing. J. Immunol. Methods 294, 89 .

Schwartz, D.A., 2002. TLR4 and LPS hyporesponsiveness in humans. Int. J. Hyg. Environ. Health 205, 221.

Schwartz, D.A., Thorne, P.S., Yagla, S.J., Burmeister, L.F., Olenchock, S.A., Watt, J.L., Quinn, T.J., 1995. The role of endotoxin in grain dust-induced lung disease. Am. J. Respir. Crit. Care Med. 152, 603. 